Historia y comunicación social

ISSN-e 1988-3056

https://dx.doi.org/10.5209/hics.66296

\title{
Susceptibilidad cognitiva a las falsas informaciones
}

\author{
Rubén Sanz Blasco ${ }^{1}$; Cristina Carro de Francisco ${ }^{2}$
}

Recibido: 29 de abril de 2019. / Aceptado: 24 de septiembre de 2019.

Resumen. Las falsas informaciones a través de los medios de comunicación no son un fenómeno novedoso. Sin embargo, el acceso a internet y la configuración y el uso de herramientas tan útiles como las redes sociales potencian la polarización y la confrontación, primando un análisis emocional e impreciso de los hechos. Se presenta un trabajo de revisión teórica que tiene como objetivo fundamental detallar los principales mecanismos psicológicos que operan en el procesamiento de las falsas informaciones centrándonos de un modo específico en la hipótesis del razonamiento motivado, los sesgos cognitivos y la teoría del proceso dual.

Palabras clave: Fake News; Sesgos Cognitivos; Revisión Teórica; Teoría del Procesamiento Dual; Razonamiento motivado.

\section{[en] Cognitive susceptibility to false information}

Abstract. False informations through the media are not a novel phenomenon. However, access to Internet, its settings, and a usage of useful tools such as social media promote the polarization and comparison, taking precedence an emotional and ambiguous analysis of facts. A theoretical review is presented with the main goal to detail the principal psychological mechanisms that operate in the processing of false information, focusing in a specific way in the motivated reasoning hypothesis, cognitive bias and dual-process theory.

Key Words: Fake News; Cognitive Bias; Theoric Review; Dual Process Theory; motivated reasoning.

Sumario: 1. Introducción 2. "Estado de la cuestión”; 3. Metodología; 4. ¿Por qué son tan sólidas las falsas informaciones?; 4.1. Teoría del razonamiento motivado; 4.2. Teoría del procesamiento dual; 4.3. Heurísticos y sesgos cognitivos; 4.3.1. Heurístico de representatividad; 4.3.2. Heurístico de disponibilidad; 4.3.3. Anclaje y ajuste; 4.3.4. Efecto de negación de precedentes; 4.3.5. Efecto de subirse al carro o efecto bandwagon; 4.3.6. Sesgo de proyección; 4.3.7. Sesgo de simetría; 5. Conclusiones; 6. Referencias bibliográficas.

Cómo citar: Sanz Blasco, R.; Carro de Francisco, C. (2019). Susceptibilidad cognitiva a las falsas informaciones, en Historia y comunicación social 24 (2), 521-531.

\footnotetext{
$1 \quad$ Universidad Complutense de Madrid. rubesanz@ucm.es

2 Universidad Complutense de Madrid. criscarro@cop.es
} 


\section{Introducción}

El rápido acceso a los contenidos periodísticos a través de internet, su facilidad de edición junto con el uso creciente de las redes sociales, han cambiado nuestros hábitos de consumo a la hora de acceder a la información, potenciando la proliferación de falsas noticias. Sin embargo, el fenómeno de las falsas informaciones no es novedoso. Realizando un breve recorrido histórico, encontramos en el año 1835 una publicación de seis entregas en el diario neoyorkino The Sun alertando a la población sobre la existencia de vida en la Luna (Allcott y Gentzkow, 2017). Otro hito histórico fue la famosa dramatización sobre la novela "La Guerra de los Mundos", que un joven Orson Wells realizó en el estudio de la emisora CBS la noche del 30 de octubre de 1938. La lectura sembró el pánico a millones de radioyentes estadounidenses que creyeron que una lluvia de meteoritos que contenía en su interior naves alienígenas estaba llegando a las calles de Nueva York y Nueva Jersey para someter al planeta. Desde finales del siglo XX conocemos la existencia de otro tipo de campañas de desinformación a gran escala relacionadas con tópicos tan diversos como son el cambio climático, las vacunas, los alimentos, la nutrición, el origen de la vida, la salud, las armas, medicamentos genéricos, el origen y la curación de enfermedades, la energía nuclear o el impacto de la inmigración (Parra y Oliveira, 2018).

Aunque sin duda, el momento más relevante del fenómeno de las falsas informaciones surgió a raíz de las elecciones norteamericanas ganadas por Donald Trump en el año 2016. Algunos comentaristas incluso han sugerido que la difusión de información incorrecta desempeñó un papel fundamental en el resultado final de la elección (para una revisión al respecto véase Parkinson, 2016; Read, 2016). Una de las numerosas investigaciones realizadas a partir de estas últimas, confirmaba que durante la campaña electoral se generaron un total de 115 noticias falsas favorables al actual presidente de Estados Unidos que se compartieron en Facebook un total de 30 millones de veces, frente a las 41 en beneficio de Hillary Clinton compartidas en 7,6 millones de ocasiones (Allcott y Gentzkow, 2017). Pero ¿a qué se refiere el término Fake News?. Recientemente ha salido publicado el libro "Fake News: la nueva arma de destrucción masiva" en el que su autor, David Alandete (2019), define el término como aquellas noticias con datos erróneos, exagerados o manipulados, que pervierten el oficio del periodismo con una finalidad política. Lazer et al. (2018: 1094) hace referencia a:

Información fabricada que imita el contenido de los medios de comunicación en forma, pero no en el proceso o la intención de la organización. Carecen de normas y procesos editoriales para eliminar lo falso a favor de lo verdadero. Las noticias falsas son, por lo tanto, un subgénero de la categoría más amplia de información errónea, de información incorrecta sobre el estado del mundo.

Existe una razón fundamental por lo que las falsas noticias prosperan: nuestro funcionamiento cerebral y un modo de procesar la información perezoso, emocional y cargado de errores cognitivos. ¿Por qué nos las creemos?, ¿Por qué nos resultan tan sólidas?, ¿Qué factores cognitivos hacen que tendamos a analizar la información de un modo acrítico resultándonos complicado protegernos y rechazar las falsas informaciones?. El presente trabajo tiene como objetivo responder a estas cuestiones y detallar los principales mecanismos cognitivos que operan en el procesamiento de las falsas informaciones. 


\section{2. "Estado de la cuestión"}

En el año 2017 el Oxford English Dictionary seleccionó el neologismo como palabra del año tras el aumento de su uso un 365\% en los últimos 12 meses, aunque un año antes ya lo hizo en Australia el Macquarie Dictionary (Bolton y Yaxley, 2017). Un dato empírico que denota el creciente interés por conceptos tales como noticias falsas o posverdad es el aumento exponencial de la producción científica sobre estos tópicos a raíz de las elecciones norteamericanas del año 2016 mencionadas con anterioridad. Una búsqueda realizada sobre ambos conceptos en la plataforma Web of Science (WOS) el 9 de agosto de 2018 refleja la evolución del fenómeno. En particular, el número de artículos de revistas fue de 150 (Parra y Oliveira, 2018).

Considerando su crecimiento exponencial y los efectos que las falsas informaciones pueden generar en la sociedad, es importante conocer los factores psicológicos que se ven implicados.

\section{Metodología}

Se ha realizado una revisión teórica en las bases de datos científicas ScienceDirect, PsycInfo, y Pubmed de la National Library of Medicine (NLM), incluyendo artículos publicados en español y en inglés y empleando las palabras clave "fake news", "cognitive bias" "social media" y el operador booleano AND.

Debido a la escasez de resultados encontrados, se llevó a cabo una nueva búsqueda en la que el descriptor en el campo título y abstract fue: "fake news".

Los artículos se filtraron según los siguientes criterios de inclusión: artículos ajustados de un modo específico al tópico de estudio, artículos con rigor metodológico y publicados en revistas científicas de reconocido prestigio, no se incluyen resúmenes de congresos y la búsqueda no ha sido acotada ateniendo a un criterio temporal.

\section{4. ¿Por qué son tan sólidas las falsas informaciones?}

Como hemos mencionado en líneas anteriores, con independencia de los intereses de diferente índole que hacen que proliferen las falsas informaciones, nuestro funcionamiento cognitivo hace que nos resulte complicado protegernos de las mismas. Explicaremos esta vulnerabilidad psicológica a las falsas noticias atendiendo fundamentalmente a la hipótesis del razonamiento motivado, la teoría del procesamiento dual y los principales errores o sesgos cognitivos que se dan en el procesamiento de la información.

\subsection{Teoría del razonamiento motivado}

Una de las teorías más ampliamente aceptada por la comunidad científica es la del razonamiento motivado que sostiene que nuestras motivaciones forman y configuran las informaciones que recibimos para que encajen con nuestras creencias, opiniones o gustos, realizando un análisis emocional de la información entrante. Es el proceso que lleva a las personas a confirmar sus creencias, ignorando los datos y hechos 
que las contradicen debido a la implicación de las emociones en los razonamientos y decisiones aparentemente racionales. Tenderíamos a procesar la información de manera que encaje con algún objetivo predeterminado explicando por qué pensamos que llevamos la razón incluso cuando estamos equivocados.

De este modo, el ser humano se muestra menos minucioso examinando evidencias congruentes con sus creencias y, sin embargo, busca el error en las informaciones y opiniones que son contrarias a éstas. A modo de ejemplo, los individuos están más inclinados a apoyar a un candidato político de su partido cuando se les presenta información negativa sobre éste (Redlawsk et al., 2010) y, por el contrario, debaten enérgicamente los argumentos que son inconsistentes con su ideología política (Strickland et al., 2011).

Si nos referimos a las falsas informaciones en el ámbito de la política, los efectos del razonamiento motivado explican por qué las afirmaciones ficticias reciben tanta aceptación en las redes sociales. Es decir, los individuos pueden ser más vulnerables a noticias falsas que sean congruentes con su ideología política (Pennycook y Rand, 2018).

\subsection{Teoría del procesamiento dual}

La teoría del procesamiento dual plantea dos sistemas diferenciados para explicar el razonamiento y pensamiento humano. Ambos procesos presentan diferencias en: aspectos funcionales, velocidad de procesamiento, acceso a la conciencia y capacidad computacional o de recursos que demandan (Seoane et al., 2017).

Por un lado, la cognición humana se caracteriza por presentar procesos automáticos e intuitivos (Sistema 1) y, por otro, procesos deliberativos y analíticos (Sistema 2) (De Neys, 2012; Evans y Stanovich, 2013; Kahneman, 2012; Pennycook et al., 2015). De este modo, el Sistema 1 realiza una interpretación coherente de lo que sucede en nuestro mundo en cualquier instante operando automáticamente. Es un sistema rápido, intuitivo que requiere de un mínimo esfuerzo y control voluntario (p.e. detectar que un objeto se encuentra más alejado que otro, detectar el enfado en una voz, entender frases sencillas, responder a $3+3=$ ?, completar la expresión "a quién madruga...", etc).

Las capacidades del Sistema 1 incluyen destrezas innatas que compartimos con otros animales. Nacemos preparados para percibir el mundo que nos rodea y evitar las amenazas, reconocer objetos, orientar la atención, etc., haciendo asociaciones entre ideas (¿cuál es la capital de Italia?). (Kahneman, 2012).

Por el contrario, el Sistema 2 enfoca su atención en las actividades mentales que se lo exigen. Está asociado a la elección y la concentración, es reflexivo, requiere esfuerzo, es lento, serial, consciente, reglado y deductivo (p.e. realizar un cálculo mental complejo, buscar una mujer pelirroja, contar las veces que aparece una vocal en una página de un texto o acordarte del nombre de una calle que a priori no recuerdas). En todas estas situaciones es necesario prestar atención, y si no se está preparado o la atención no es la adecuada, las actividades correspondientes se realizarán peor o no se llevarán a término (Kahneman, 2012). Una de las demostraciones más evidentes sobre este fenómeno lo demostraron Chabris y Simons (2011) en su libro "El gorila invisible: cómo nuestras intuiciones nos engañan". Los autores pidieron a un grupo de voluntarios que observasen un vídeo de un minuto de duración en el que unos jugadores de baloncesto se pasaban una pelota y que contasen los pases. La 
tarea requiere una elevada concentración. En un momento determinado, aparecía en la pantalla una mujer disfrazada de gorila, que se golpeaba el pecho con los puños y se iba. Sorprendentemente casi la mitad de los participantes no observaron al gorila. La tarea que debían realizar les volvió ciegos. El estudio muestra dos hechos importantes relativos a nuestra mente, por un lado, podemos estar ciegos para lo evidente debido al funcionamiento automático del sistema 1 que simplemente se concentra en las instrucciones concretas de los pases del balón y ciegos además a nuestra propia ceguera (Kahneman, 2012).

En cuanto a la interacción de los dos sistemas, Kahneman (2012) afirma que siempre están activos mientras permanecemos despiertos. El Sistema 1 actúa automáticamente y el Sistema 2 se halla normalmente en un confortable modo de mínimo esfuerzo en el que solo una fracción de su capacidad está ocupada. El Sistema 1 realiza de manera constante sugerencias al Sistema 2 en forma de impresiones, intuiciones, intenciones y sensaciones. Si cuentan con la aprobación del Sistema 2 , dichas impresiones se tornan creencias y los impulsos en acciones voluntarias. Asimismo, el Sistema 2 tiene cierta capacidad para cambiar el modo de trabajar del Sistema 1 programando funciones normalmente automáticas de la atención y la memoria.

A modo de ejemplo práctico, considere el siguiente problema extraído de la Prueba de Reflexión Cognitiva (CRT) de Frederick (2005):

Un bate y una pelota cuestan $1.10 €$. El bate cuesta $1 €$ más que la pelota. ¿Cuánto cuesta la pelota? El problema elicita una respuesta rápida e intuitiva (10 céntimos). Al reflexionar, nos percatamos de que es errónea (si la pelota costara 10 céntimos, el bate tendría que costar 1,10€ y sumarían un total de 1,20€), siendo la respuesta correcta 5 céntimos. La respuesta intuitiva incorrecta basada en el sistema 1 dada por el 65\% de individuos según el estudio de Pennycook et al., (2016b) muestra un fallo generalizado en los procesos de razonamiento reflexivo (Pennycook y Ross, 2016a).

En el contexto de las falsas noticias, el estudio de Pennycook y Rand (2018), demuestra por qué los individuos creen en los titulares de noticias falsas. Para ello, administraron a 3.446 participantes una prueba CRT que medía la tendencia a desafiar las reacciones intuitivas. Posteriormente, les mostraron publicaciones en Facebook con tres tipos de titulares: noticias reales, noticias falsas que reforzaban su ideología política, y noticias falsas que la contradecían. A cada participante se le pidió que calificara la precisión de cada una de las tres historias. Los resultados ponen de manifiesto que los individuos más reflexivos eran más capaces de diferenciar entre noticias reales y falsas, independientemente de si el titular estaba de acuerdo con su ideología política. Sin embargo, las personas menos reflexivas eran más propensas a creer todo lo que leían. Por tanto, por un lado, el empleo del pensamiento analítico (Sistema 2) predice la capacidad de discernir entre noticias falsas y reales, independientemente de si es coherente o inconsistente con la ideología política de una persona (Pennycook y Rand, 2018) pero no es nuestro modo habitual de procesar la información. Siguiendo un principio de economía cognitiva nuestro cerebro, de manera natural, trata de pensar con el menor coste o esfuerzo posible mediante el uso del Sistema 1, tendemos a suprimir las dudas e ignorar la ambigüedad de un modo rápido. En definitiva, busca reducir los conflictos o disonancias cognitivas, favoreciendo aque- 
llas informaciones que concuerdan con las propias creencias sin realizar en muchas ocasiones un análisis racional de los hechos.

Por si fuera poco, el ecosistema digital está configurado para adaptarse a nuestro funcionamiento cognitivo. Uno de los principales problemas del consumo de noticias a través de las plataformas digitales es que son gratuitas. Debido a esto la supervivencia de muchos medios dependen de los ingresos que se obtienen de la publicidad. Es necesario que se vean muchos anuncios para poder subsistir lo que lleva a miles de individuos a producir contenido y noticias a gran escala, tratando de luchar por un proceso cognitivo limitado como lo es nuestra atención. En definitiva, los medios de comunicación a través de internet se encuentran al servicio de la publicidad generándose una gran cantidad de contenidos con el fin de insertar anuncios publicitarios. Debido a que generar contenido de calidad con información contrastada es muy costoso, resulta mucho más atractivo, fácil y económico la generación de falsas noticias. En un mundo con una gran sobrecarga informativa, la lucha por nuestra atención no reportaría tantos beneficios si el cerebro no siguiera unos determinados patrones de funcionamiento como los analizados y, esencialmente, por la actuación del denominado sesgo de confirmación. Para no realizar demasiado esfuerzo ante cada noticia, evaluando de un modo consciente y analítico su veracidad, se tiende a sobreestimar el contenido que encaja y confirma nuestras ideas o creencias en detrimento de aquellas informaciones que no se adaptan a nuestras expectativas, a lo que pensamos o creemos o que simplemente no nos generan afecto positivo.

Además, tendemos a seguir a usuarios, leer periódicos o consumir contenido que encaja con nuestras ideas preconcebidas, ignorando al resto de la información. El clickbait se aprovecha de nuestro sesgo de confirmación. Mediante el uso de algoritmos se ofrece un contenido adaptado a las preferencias de búsqueda y consumo, generándose la falacia de que la mayoría de personas piensan como uno mismo. Si el contenido se adapta mejor, se obtienen más clicks y más tiempo se invertirá leyendo, lo que en última instancia se traduce en beneficios económicos. Es decir, no sólo el cerebro trata de evitar realizar un análisis racional de los hechos para minimizar un elevado coste cognitivo, sino que las propias plataformas se aprovechan de este hecho realizando el trabajo de criba por nosotros. En muchas ocasiones no se tendrá la ocasión de tener que ignorar o suprimir la información que es contraria a nuestras ideas o creencias, hecho que de manera natural nuestro Sistema 1 haría por si mismo, ya que ni tan siquiera tendremos la ocasión de poderla ver.

\subsection{Heurísticos y sesgos cognitivos}

Las limitaciones de nuestros procesos cognitivos, la falta de información cuando nos enfrentamos a situaciones desconocidas y la incertidumbre hacen que nuestro cerebro recurra a heurísticos. Dichos heurísticos hacen referencia a reglas generales y poco definidas que funcionan como "atajos mentales" para resolver problemas complejos. Resultan útiles para disminuir la sobrecarga cognitiva y nos permiten ahorrar recursos mentales. No obstante, aunque aportan vías para simplificar los problemas, nos pueden llevar a cometer errores o sesgos cognitivos ya que nos llevan a percibir la realidad a través de juicios inexactos y conclusiones ilógicas. Aunque no son los únicos, a continuación, se detallarán los heurísticos y sesgos que impulsan la creencia en las falsas informaciones (ver tabla 1). 


\subsubsection{Heurístico de representatividad}

Se relaciona con el grado en que un objeto o suceso comparte las características de un conjunto determinado de objetos o sucesos. Es decir, se trata de juzgar la probabilidad de que una persona, acción o sujeto pertenezca a un conjunto o categoría fijándose en la semejanza entre el mismo y los miembros del grupo. Se trata de una manera de razonar que permite dar respuestas rápidas a las situaciones habituales, permitiendo un importante ahorro cognitivo. Un ejemplo de este heurístico serían los prejuicios sociales emitidos a las personas por razón de raza, religión, sexo, etc.

\subsubsection{Heurístico de disponibilidad}

Consiste en basar nuestras creencias y decisiones en la información que aparece en primer lugar en nuestra mente creyendo que lo más disponible es lo más frecuente o probable, cuando sólo podemos acceder a los datos más recientes o a los más frecuentes y en ocasiones éstos son imprecisos o vagos. Debido a que los juicios de probabilidad se producen ante la ausencia de datos objetivos, tendemos a recurrir a los datos que podemos recuperar de la memoria. Es decir, los individuos evaluamos la probabilidad de un suceso por la mayor o menor facilidad con que ese recuerdo se activa en nuestra mente. A modo de ejemplo, podríamos juzgar como más peligroso viajar en avión que en automóvil debido a que los accidentes aéreos tienen más cobertura mediática y, por tanto, están más disponibles en nuestra memoria.

\subsubsection{Anclaje y ajuste}

Los individuos emitimos juicios a partir de un valor inicial que se va ajustando hasta obtener la respuesta definitiva. Dicho valor puede estar contenido en la misma formulación del problema o ser el resultado de un cálculo parcial. Estos ajustes no son suficientes ya que si partimos de diferentes puntos obtendremos distintas estimaciones que estarán sesgadas por los valores iniciales. El valor se ajusta según se va añadiendo nueva información.

En particular, el efecto de anclaje en las falsas noticias haría referencia a que damos mayor peso al primer argumento, noticia o evidencia que nos llega a la mente. A partir de ese ancla se genera el juicio o se toman decisiones. Dicho de otro modo, las informaciones que llegan en primer lugar y que son repetidas se archivan en nuestra memoria como más ciertas que la aparición de nueva información o que el desmentido. Esto se debe a un error en la clasificación de los recuerdos que hace que cuando creemos una noticia y nos "anclamos" en un determinado contenido con el paso del tiempo el cerebro atribuye que se ha obtenido de una fuente fiable.

\subsubsection{Efecto de negación de precedentes}

Hace referencia a la tendencia de algunas personas a evitar añadir probabilidades o eventos precedentes bien conocidos que podrían ser relevantes en una decisión a tomar o en la conclusión realizada. Siguiendo con el ejemplo utilizado anteriormente, los datos nos dicen que probabilísticamente viajar en coche es más peligroso que hacerlo en avión. Sin embargo, muchas personas no incorporan esta probabilidad 
objetiva a sus razonamientos a la hora de tener que decidirse por uno de los dos medios de transporte. Dicho de otro modo, cuando razonamos y tratamos de mantener una posición o argumento, tendemos a negar y no incorporar datos conocidos que podrían cambiar la decisión u opinión conformada.

\subsubsection{Efecto de subirse al carro o efecto bandwagon}

Se refiere a que los individuos nos comportamos y pensamos multitud de cosas simplemente por la opinión que tiene nuestro ambiente o contexto. Si leemos muchas noticias en la misma dirección o nos rodeamos de personas que piensan igual nos costará ser críticos y opinar lo contrario.

\subsubsection{Sesgo de proyección}

También denominado el sesgo del falso consenso, hace referencia a la tendencia a interpretar que los demás tienen las mismas creencias, pensamientos, razonamientos o valores que uno mismo.

\subsubsection{Sesgo de simetría}

Dicho sesgo es la tendencia inconsciente a considerar como más ciertos y sólidos los argumentos y razonamientos en los que exista una dicotomía clara. La existencia de una simetría, aunque hace al argumento considerado más atractivo para nuestro sistema cognitivo, no implica la veracidad del mismo (p.e. preferencias políticas).

Se presentan en la tabla 1 otros sesgos y heurísticos:

\begin{tabular}{|c|c|}
\hline Sesgo & Definición \\
\hline Sesgo de Autoridad & $\begin{array}{l}\text { Se refiere a que las informaciones con independencia de su veraci- } \\
\text { dad son más persuasivas según el peso de la autoridad del informan- } \\
\text { te. Cuanto más confiemos en la persona o medio que nos informa, } \\
\text { menos críticos seremos en el análisis de la información (p.e. leer un } \\
\text { artículo y creer en la información que contiene porque lo escribe un } \\
\text { investigador de prestigio). }\end{array}$ \\
\hline Efecto Halo & $\begin{array}{l}\text { Hace referencia a la realización de una generalización a partir de una } \\
\text { sola característica o rasgo observado de un individuo o de un objeto } \\
\text { (p.e. ver a alguien que es apuesto y asumir que su manera de ser nos } \\
\text { resultará igual de atractiva). }\end{array}$ \\
\hline $\begin{array}{l}\text { Efecto de Encuadre } \\
\text { (framing) }\end{array}$ & $\begin{array}{l}\text { Se refiere a que las conclusiones que extraemos de una información } \\
\text { y las reacciones ante diferentes decisiones varían según se nos pre- } \\
\text { senten los datos (p.e. una bebida tiene un } 7 \% \text { de zumo natural. Una } \\
\text { bebida contiene un } 93 \% \text { de sustancias colorantes, conservantes, etc). }\end{array}$ \\
\hline $\begin{array}{l}\text { Sesgo de debilidad } \\
\text { y fortaleza }\end{array}$ & $\begin{array}{l}\text { Es la tendencia de las personas a juzgar como más permisibles o } \\
\text { creer con mayor fuerza los comportamientos y los argumentos de } \\
\text { las partes más débiles o de las más fuertes (p.e. Con independencia } \\
\text { del discurso, dar la razón al débil o al fuerte por el simple hecho de } \\
\text { serlo). }\end{array}$ \\
\hline
\end{tabular}




\begin{tabular}{|c|c|}
\hline $\begin{array}{l}\text { Observación } \\
\text { Selectiva o Ilusión } \\
\text { de Frecuencia }\end{array}$ & $\begin{array}{l}\text { Hace referencia a que los individuos observamos mucho más a lo } \\
\text { que nos influye de forma directa y le atribuimos una mayor frecuen- } \\
\text { cia de aparición e importancia de la que realmente tiene (p.e. un } \\
\text { amigo nos recomienda un libro y desde ese momento lo vemos en } \\
\text { anuncios, en pasajeros leyéndolo en el autobús, etc. Pensaremos que } \\
\text { ese libro es mucho más leído que otros, no porque nos hayamos in- } \\
\text { formado de sus datos de venta sino porque al atenderlo más nos pa- } \\
\text { rece mucho más frecuente). }\end{array}$ \\
\hline Punto Ciego & $\begin{array}{l}\text { Nos demuestra que los individuos tenemos escasa conciencia de } \\
\text { nuestros propios sesgos. Los identificamos fácilmente en los demás, } \\
\text { pero pasan desapercibidos cuando nosotros caemos en uno, lo que } \\
\text { nos hace ser menos críticos (p.e. vemos la paja en el ojo ajeno, pero } \\
\text { no la viga en el propio). }\end{array}$ \\
\hline $\begin{array}{l}\text { Falacia Ad } \\
\text { Verucundiam }\end{array}$ & $\begin{array}{l}\text { Consiste en defender una conclusión apelando a alguien que se con- } \\
\text { sidera una autoridad en la materia, sin aportar argumentos que la } \\
\text { justifiquen. (p.e. esto es así porque lo he leído en internet). }\end{array}$ \\
\hline $\begin{array}{l}\text { Falacia Ad } \\
\text { Hominem }\end{array}$ & $\begin{array}{l}\text { Se refiere a pretender rebatir el razonamiento de otra persona o de- } \\
\text { mostrar la falsedad de la conclusión a la que ha llegado tratando } \\
\text { de desacreditar a quien la defiende. Un ejemplo de índole política } \\
\text { sería cuando un candidato le dice a otro: ¿Cómo puede hablar de } \\
\text { corrupción si cuando ustedes gobernaban cada día descubríamos un } \\
\text { nuevo caso de corrupción?. }\end{array}$ \\
\hline $\begin{array}{l}\text { Falacia Ad } \\
\text { Ignorantiam }\end{array}$ & $\begin{array}{l}\text { Consiste en la defensa de que algo es definitivamente verdadero o } \\
\text { falso únicamente porque no podemos demostrar lo contrario lo que } \\
\text { científicamente resulta incorrecto (p.e. no se ha probado totalmente } \\
\text { determinada teoría, luego esta teoría es falsa). }\end{array}$ \\
\hline Generalización & $\begin{array}{l}\text { Se trata de inferir una conclusión general a partir de unos pocos ca- } \\
\text { sos particulares o incidentes aislados (p.e. un miembro de un deter- } \\
\text { minado partido político es acusado de malversación. Por tanto, todo } \\
\text { el partido es corrupto). }\end{array}$ \\
\hline $\begin{array}{l}\text { Pendiente } \\
\text { Resbaladiza }\end{array}$ & $\begin{array}{l}\text { Hace referencia a la tendencia a llegar a conclusiones que no parten } \\
\text { de las premisas precedentes (p.e. en un partido político han descu- } \\
\text { bierto que uno de sus miembros ha prevaricado. Seguro que además } \\
\text { ha hecho cosas peores). }\end{array}$ \\
\hline
\end{tabular}

Tabla 1. Otros errores, sesgos o falacias que pueden relacionarse con las falsas informaciones. "Elaboración propia"

\section{Conclusiones}

A lo largo del presente trabajo hemos tratado de realizar un recorrido por los aspectos fundamentales acerca de los procesos cognitivos que operan en el procesamiento de la información en general y de las falsas informaciones en particular.

Proporcionar informaciones falsas con la intención de engañar al público se ha convertido en un problema globalizado que se ha visto reforzado por la omnipresencia de Internet, la aparición de las redes sociales y la posibilidad de viralización (Parra y Oliveira, 2018). Las informaciones falsas se convierten en un fenómeno que tiende a prosperar debido a un mundo digital mediático y publicitario que lo potencia 
con escasos filtros de calidad y de revisión de la información, pero especialmente porque se adapta y se aprovecha del modo en el que sentimos y pensamos.

Debido a nuestro funcionamiento cerebral sabemos que las falsas informaciones se procesan fácilmente y desacreditarlas resulta complicado. Como hemos podido comprobar, limitarnos a decir que una información es falsa no es efectivo. Siguiendo la Teoría del Razonamiento Motivado, hemos señalado algunos estudios recientes en los que se ha puesto de manifiesto que una de las explicaciones de su crecimiento es que las personas tienden a convencerse a sí mismas de que incluso las noticias inverosímiles son correctas si son coherentes con sus creencias e ideologías (Beck, 2017; Calvert, 2017; Kahan, 2017; Singal, 2017). De manera complementaria, el ser humano tiende a economizar recursos cognitivos, empleando el Sistema 1 de pensamiento, de carácter rápido e intuitivo, lo que nos lleva en ocasiones a generar conclusiones precipitadas y erróneas. Por contra, diversos estudios han demostrado que el razonamiento analítico, empleando el Sistema 2 de pensamiento, aunque no es nuestro sistema primario o habitual de procesar la información, está vinculado a la anulación de las creencias en una gran variedad de ámbitos o, dicho de otro modo, nos protege de los rumores y de las falsas informaciones (para una revisión, véase Pennycook, et al., 2015). Por otro lado, resulta importante conocer las limitaciones de nuestros procesos cognitivos (atención, memoria, etc.), que hacen que a nuestro cerebro no le quede más remedio que recurrir a heurísticos o atajos que si bien resultan de gran utilidad en la descarga de los recursos mentales también provocan la comisión de errores en el procesamiento de la información entrante, especialmente si tiene una elevada carga emocional.

En última instancia, conociendo nuestro sistema cerebral y sabiendo que éste funciona bajo un principio de economía cognitiva, con el objetivo de protegernos de las falsas informaciones, resulta relevante analizar la noticia en profundidad, especialmente si es importante o nos atañe, huir de los titulares y prestar atención a lo que no se cuenta. Además, debemos tener presente que los sesgos del informado son los mismos que tiene el informante. Recordar que en la información política o ideológica nuestros sesgos se incrementan debido al componente emotivo y al sentido de pertenencia.

Para finalizar, con el objetivo de reducir las falsas informaciones, el meta-análisis de Chan et al. (2017) propone tres implicaciones prácticas para los responsables de la opinión pública, comunicadores y editores, a saber: informar facilitando la verificación de la información así como dar pie a la argumentación, disminuir la posibilidad de crear argumentos que estén a favor de la desinformación y, por último, corregir la desinformación con nueva información detallada.

\section{Referencias bibliográficas}

Alandete, D. (2019). Fake News: la nueva arma de destrucción masiva, Barcelona, Deuesto S.A. Ediciones.

Allcott, H. y Gentzkow, M. (2017). "Social Media and Fake News in the 2016 Election". NBER Working Paper No. 23098. Retrieved from <http://www.nber.org/papers/ w23089>.

Beck, J. (2017). “This article won't change your mind: The fact on why facts alone can't fight false beliefs". The Atlantic Retrieved from https://www.theatlantic.com/science/ archive/2017/03/this-article-wont-change-your-mind/519093/. 
Bolton, D. M. y Yaxley, J. (2017). "Fake news and clickbait-natural enemies of evidence-based medicine". BJU International, 119, pp. 8-9.

Calvert, D. (2017). "The Psychology Behind Fake News". Retrieved August 2, 2017, from $<$ https://insight.kellogg.northwestern.edu/article/the-psychology-behind- fake-news $>$.

Chabris, C y Simons D. (2011). "El gorila invisible: cómo nuestras intuiciones nos engañan". Barcelona, RBA Libros.

Chan, M.P. Jones, C. Jamieson, K y Albarracín, D. (2017). “Debunking: A Meta-Analysis of the Psychological Efficacy of Messages Coutering Misinformation". Psychological Science, 28(11), pp. 1531-1546.

De Neys, W (2012). "Bias and conflict: A case for logical intuitions". Perspectives on Psychological Science, 7(1), pp. 28-38.

Evans, J. y Stanovich, K. (2013). "Dual-process theories of higher cognition: Advancing the debate”. Perspectives on Psychological Science, 8(3), pp. 223-241.

Frederick, S. (2005). "Cognitive reflection and decision making”. Journal of Economic Perspectives, 19(4), pp. 25-42.

Kahan, D. (2017). "Misconceptions, misinformation, and the logic of identity protective cognition". SSRN Electronic Journal, 164 Disponible en; http://dx.doi.org/10.2139/ ssrn.2973067.

Kahneman, D. (2012). Pensar rápido, pensar despacio, Barcelona, Penguin Random House Grupo Editorial, S.A.U.

Lazer, D. Baum, M. Benkler, J. Berinsky, A. Greenhill, K. Metzger, M. y Zittrain, J. (2018). "The science of fake news". Science, 9, pp. 1094-1096.

Parkinson, H. J. (2016). "Click and Elect: How fake news helped Donald Trump win a real election". The Guardian (November 14).

Parra, P., y Oliveira, L. (2018). "Fake news: una revisión sistemática de la literatura”. Observatorio Journal, Special Issue, pp. 054-078.

Pennycook, G. Fugelsang, J. y Koehler, D. (2015). "What makes us think? A three- stage dual-process model of analytic engagement". Cognitive Psychology, 80, pp. 34-72.

Pennycook, G. y Rand, D. (2018). "Lazy, not biased: Susceptibility to partisian fake news is better explained by lack of reasoning tan by motivated reasoning". Cognition. Disponible en: https://doi.org/10.1016/j.cognition.2018.06.011.

Pennycook, G. y Ross, R. (2016a). "Commentary on: Cognitive reflection vs. calculation in decision making”. Frontiers in Psychology, 7, pp. 9.

Pennycook, G. Ross, R. Koehler, D. y Fugelsang, J. (2016b). "Atheists and agnostics are more reflective than religious believers: Four empirical studies and a meta-analysis". Plos One, 11(4), pp. 1-18.

Read, M. (2016). "Donald Trump won because of facebook". New York Magazine (November 9).

Redlawsk, D. Civettini, A. y Emmerson, K. (2010). “The affective tipping point: Do motivated reasoners ever “Get It”?". Political Psychology, 31(4), pp. 563-593.

Seoane, G. Valiliña, M. Rodríguez, M. Martín, M. y Ferraces, M. (2007). "Diferencias individuales en razonamiento hipotético-deductivo: importancia de la flexibilidad y de las habilidades cognitivas". Psicothema, 19(2), pp. 206-211.

Singal, J. (2017). "This is a great psychological framework for understanding how fake news spreads". New York Magazine. Retrieved from < https://www.thecut. com/2017/01/a-great-psychological-framework-for-understanding-fake-news.html >.

Strickland, A. Taber, C. y Lodge, M. (2011). "Motivated reasoning and public opinion. Journal of Health Politics", Policy and Law, 36(6), pp. 89-122. 